\title{
Numerical Investigation on the Flow Field Produced by Oscillating Foils
}

\author{
Han-Chieh Chiu ${ }^{1}$, Ren-Horn Shieh ${ }^{1}$, Yu-Jen Chiu ${ }^{1}$, Jer-Huan Jang ${ }^{2,3, a}$, and Cheng-Ru Yu ${ }^{2}$ \\ ${ }^{1}$ Department of Mechanical Engineering, Taipei City University of Science and Technology, Taipei 112, Taiwan \\ ${ }^{2}$ Department of Mechanical Engineering, Ming Chi University of Technology, New Taipei City 243, Taiwan \\ ${ }^{3}$ Department of Mechanical Engineering, Chang Gung University, Taoyuan 333, Taiwan
}

\begin{abstract}
In present manuscript, the flow field produced by oscillating foils in a duct were under investigation numerically. The flow field was considered as an incompressible laminar transient flow. In this paper, the oscillating frequencies were 2, 4 and 6 $\mathrm{Hz}$; the span angles of the foil were $30^{\circ}, 60^{\circ}, 90^{\circ}, 120^{\circ}$, and $150^{\circ}$, respectively. The flow field produced by a particular oscillating pattern (fast forward slow backward, FFSB) has been made in comparison with that by the sinusoidal oscillating pattern. The flow field produced by dual oscillating foils was also analysed. Two foils oscillated in the same direction (in-phase) and in the opposite direction (counter-phase) were studied as well. Commercial software ANSYS-FLUENT was employed for the 2D numerical simulation. A moving grid technique was utilized in the analysis. Results present the flow average velocity increases as the oscillating frequency increases. It is also shown that the flow average velocity with span angle of $120^{\circ}$ is the largest among these cases. In addition, the flow average velocity with dual foils is better than that with a single foil.
\end{abstract}

\section{Introduction}

The progress of semiconductor industry has reduced the scale of electronic devices and increases the heat flux of heat sources. The heat dissipation rate from electronic devices requires new designs of miniaturized cooling components. Due to limitations of space in electronic devices, forced convective cooling with fans was not available. However, electronic cooling with combined conduction and free convection was hardly sufficient for electronic devices. One of the potential issues is the convective heat transfer with miniature oscillating foils. Therefore, research on the oscillation of small plate become one of the important issues for compact electronic cooling.

In cases where rotary fans are ineffective for dissipating heat, the piezoelectric fan provides a potential and reliable alternative approach to heat transfer. Piezoelectric fans can be fabricated in small sizes to fit applications that require miniature cooling modules. Toda [1, 2] first proposed the concept of piezoelectric fans for a cooling system with small size and low power consumption. He employed piezoelectric material as driven element with driving voltage of $110 \mathrm{~V}$ and the frequency of $13 \mathrm{~Hz}$. As the plate was oscillating in the resonance frequency, its amplitude would increase and the surrounding air was driven greatly. The oscillating plate could be miniaturized and therefore considered as the cooling of small electronic devices with considerable potential.

Yoo et al [3] studied the resonant frequencies for various foil designs. The resonant frequency was found to be inversely proportional to square of foil length. The maximum flow velocity close to the tip of foil can reach $3.0 \mathrm{~m} / \mathrm{sec}$ at appropriate resonant frequency. Wait et al [4] measured the amplitudes of piezoelectric fan for higher resonance modes. The energy consumption and flow field were assessed for various foil lengths and resonant frequencies. Acikailn et al. [5] observed the flow field around fan tip and investigated the heat transfer coefficient on heat source. Liu et al [6] tested the thermal performance of fans with various fan geometries and horizontal/vertical arrangements. Kimber and Garimella [7] measured various piezoelectric fans mounted perpendicular to a constant heat flux surface and studied the thermal performance across specific range of fan dimensions, vibration frequency, and amplitude. The experimental results show that vibration frequency has more significant effect on the heat transfer rate than vibration amplitude. Li et al [8] studied the effects of configuration, foil location, and heat sink design.

Fan array was a further development on this issue. Multiple fans were utilized in driving air flow and cooling system. Kimber and Garimella [9] built dual fan array to study the cooling performance on vertical surface. Ma et al. [10] designed multiple vibrating-fan cooling system actuated by combining piezoelectric force and magnetic force.

From the literatures cited above, it is found that most of the studies are investigated through experiment. Numerical analysis on the flow field induced by oscillating foil has been rarely studied. The main purpose of this paper is to investigate the air flow induced by single and dual oscillating foils. The effects of oscillating frequency, and oscillating pattern, span angle of oscillation have been studied to estimate the average velocity of the air flow.

\footnotetext{
${ }^{a}$ Corresponding author: jhjang@mail.mcut.edu.tw
} 


\section{Numerical analysis}

\subsection{Physical Model}

The physical model of the present study is shown in Figure. 1. The channel length is $400 \mathrm{~mm}$ with width of $90 \mathrm{~mm}$ and depth of 5 $\mathrm{mm}$. An oscillating foil is located $80 \mathrm{~mm}$ away from the inlet and at the centre of the channel, as shown in Figure. 1(a). The foil is rectangular and $40 \mathrm{~mm}$ in length with thickness of $1.5 \mathrm{~mm}$ and height of $4.5 \mathrm{~mm}$. The span angle of foil is $2 \theta$ with oscillation symmetric to the centreline. The span angles of the foil are $30^{\circ}, 60^{\circ}, 90^{\circ}, 120^{\circ}$, and $150^{\circ}$, respectively. In present study, dual foil oscillation is also under investigation. The second foil is located downstream to the first one. The distance between two foils is varied to study its influence on the flow. The distance between two foils are $44 \mathrm{~mm}, 50 \mathrm{~mm}, 60 \mathrm{~mm}, 70 \mathrm{~mm}$, and $80 \mathrm{~mm}$, respectively. The two foils are in the same dimension.
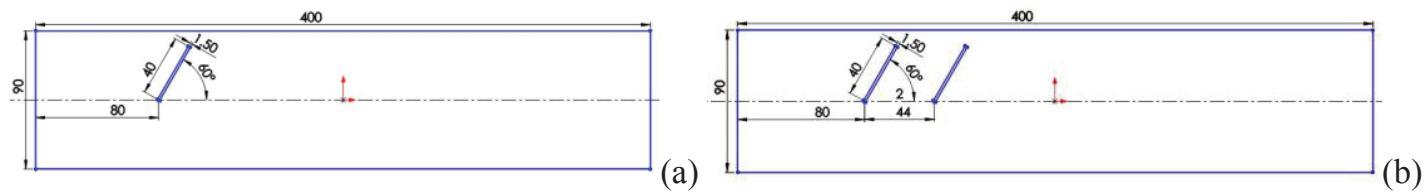

Figure 1. Two-dimensional physical model with (a) single foil; (b) dual foils.

\subsection{Analysis}

Because of the physical model, the depth of the channel is relatively small compared with its length and width, the air flow is considered as two-dimensional flow. Due to small amount of air drawn into the channel, the induced air flow is assumed to be incompressible laminar flow with constant properties in the present model. Actually, the foil would deform due to eccentric force by oscillation. However, the foil is considered rigid in order to simplify the problem.

The commercial software of ANSYS-Fluent was employed to analyse the physical 2D model. The dynamic mesh scheme was adopted in the area of foil sweeping, the meshes are defined as deformable meshes, such that they can be stretched or compressed while the foil is in motion. Therefore, the elements around oscillating foils would be stretched or compressed as shown in Figure. 2. As for the boundary conditions, oscillating foil and the wall were set as wall condition, the inlet was set as vent inlet, and the outlet was set as vent outlet. A transient flow with oscillating foils in motion for $10 \mathrm{~s}$ has been calculated. There are 20 steps for one complete cycle.

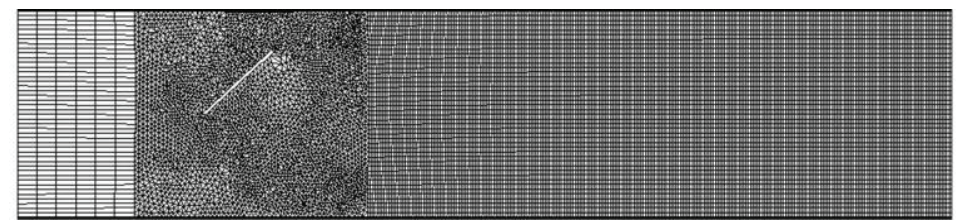

(a)
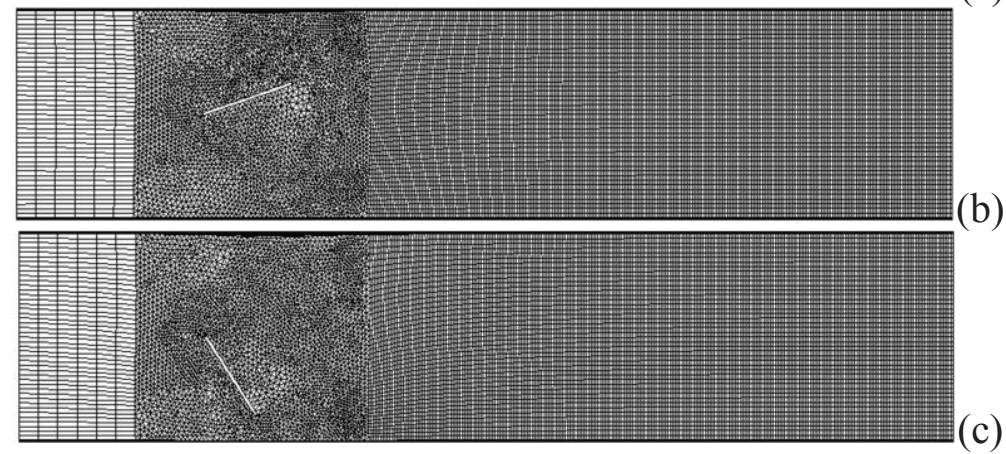

(b)

Figure 2. Sketch diagram of the dynamic meshes.

Most of the researches considered oscillating velocity symmetric to the central axis of the oscillation. However, the optimal oscillating velocity profile may not be in the sinusoidal form or a harmonic function. From the aspect of fluid dynamics, the rotating velocity should be higher as the foil is sweeping toward downstream. In other word, the rotation of foil from one end to the central line could be faster than that from the central line to the other end. In this paper, a fast forward slow backward (FFSB) oscillating pattern has been generated to investigate the effect of oscillating pattern on the induced air flow. In the FFSB pattern, as the foil rotates from one end to the central line, the angular velocity is higher; while the foil rotates from the central line to the other end, the angular velocity is lower. The oscillating pattern can be generated in the user defined function in ANSYS-Fluent. The oscillating patterns are presented in Figure. 3. 


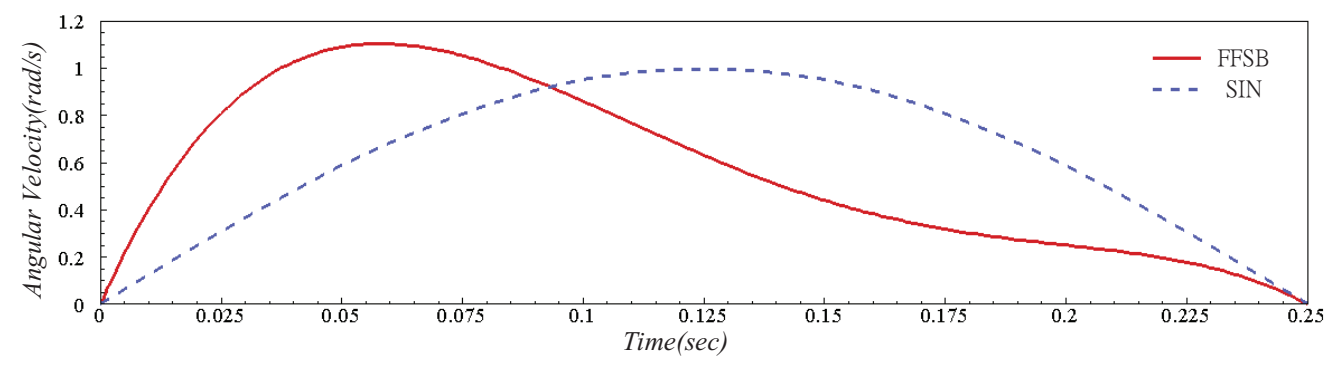

Figure 3. Oscillating pattern as a function of time for oscillating frequency of $2 \mathrm{~Hz}$.

\section{Results and Discussions}

In present study, numerical simulation on the induced air flow by oscillating foils has been conducted. Effects of oscillating frequencies, span angles, oscillating patterns, and dual foils are considered. The oscillating frequencies are 2, 4, and $6 \mathrm{~Hz}$; the span angles of the foil were $30^{\circ}, 60^{\circ}, 90^{\circ}, 120^{\circ}$, and $150^{\circ}$, respectively. The flow field produced by dual oscillating foils was also analysed. Two foils oscillated in the same direction (in-phase) and in the opposite direction (counter-phase) were studied. The distance between two foils is varied to study its influence on the flow. The distance between two foils are $44 \mathrm{~mm}, 50 \mathrm{~mm}, 60 \mathrm{~mm}$, $70 \mathrm{~mm}$, and $80 \mathrm{~mm}$, respectively.

\section{1 streamline, velocity, and pressure distributions of flow field}

Figure 4 shows the streamline diagram of the air flow with single oscillating foil at the frequency of $4 \mathrm{~Hz}$ and span angle of $120^{\circ}$. Figure 4(a) is the flow streamline as the foil is at the bottom; while Figure 4(c) is the flow streamline as the foil is at the centre. It is found that the air is drawn into the channel uniformly. In addition, vortices continuously occur towards channel outlet after the air flow passing through the oscillating foil. The corresponding velocity and pressure contours are presented in Figure. 5 and 6 , respectively. It is seen that the high velocity occurs where stream lines are dense; while the pressure is low. It is also notice that vortices move downward regularly. However, some vortices are affected between the foil and the channel walls.

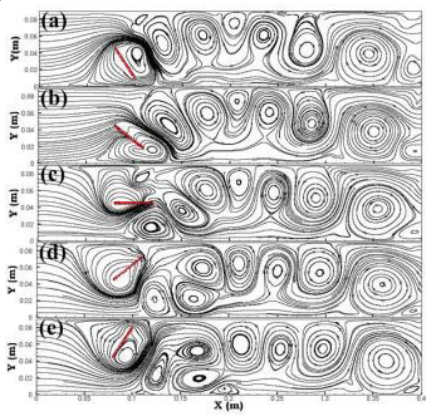

Figure4. Streamline diagram with single oscillating foil.

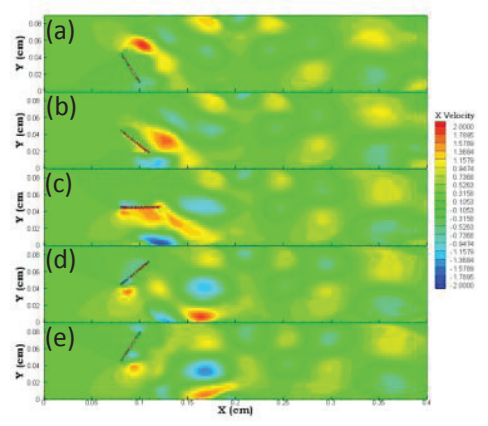

Figure 5. Velocity contour with single oscillating foil with oscillating frequency of $4 \mathrm{~Hz}$. 


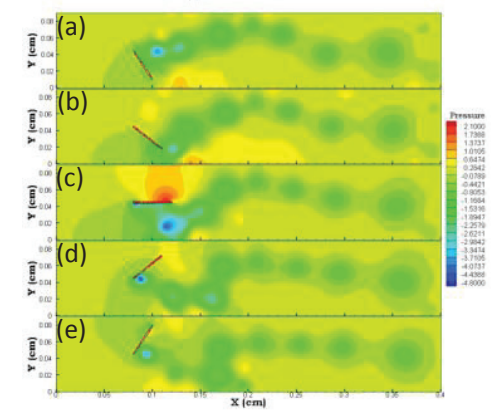

Figure6. Pressure contour with single oscillating foil with oscillating frequency of $4 \mathrm{~Hz}$.

\subsection{Effect of span angle}

The air flow induced by the oscillating foil is affected by the span angle of the foil. In this study, the effect of span angle on the averaged velocity in the channel has been investigated. The span angles are $30^{\circ}, 60^{\circ}, 90^{\circ}, 120^{\circ}$, and $150^{\circ}$, respectively. Figure 7 presents the effect of oscillating span angle on the average velocity in the channel for three oscillating frequencies. It is found that the flow average velocity increases as oscillating frequency increases at each span angle. It is also discovered that the optimum flow average velocity occurs at the span angle of $120^{\circ}$. It is observed from the numerical flow animation that the flow velocity was hindered by the channel wall, resulted in a lower flow average velocity in the channel.

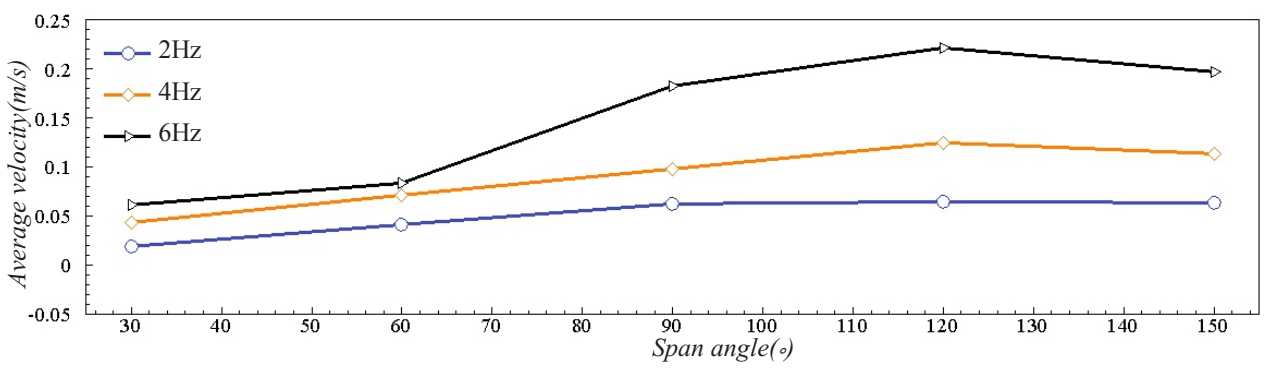

Figure 7. Effect of span angle on the flow average velocity in the channel for three oscillating frequencies.

\subsection{Effect of oscillating pattern}

Figure 8 depicts the effect of oscillating pattern on the flow average velocity in the channel for three oscillating frequencies. It is discovered that the flow average velocity with FFSB pattern is larger than that with sinusoidal pattern for three oscillating frequencies. This indicates that sinusoidal oscillating pattern is not an optimal oscillating pattern in producing air flow. This confirms the concept that a FFSB is a better oscillating pattern for producing air flow with oscillating foil.

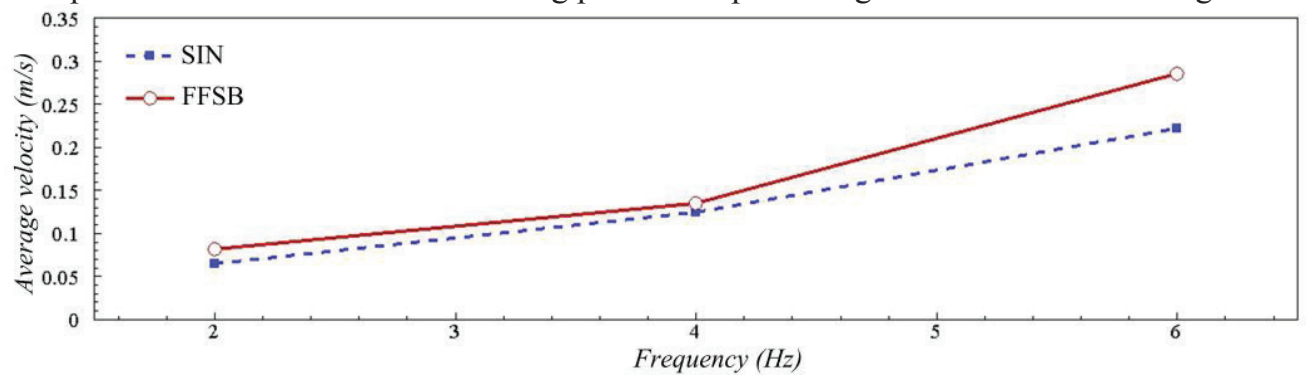

Figure 8. Effect of oscillating pattern on the flow average velocity in the channel for three oscillating frequencies.

\subsection{Dual oscillating foils}

In the present study, the air flow induced by dual foil oscillation was studied as well. In addition, two foils oscillated in the same direction (in-phase) and in the opposite direction (counter-phase) were studied. Figure 9 presents the effect of distance between two foils on the flow average velocity in the channel with three oscillating frequencies for both in-phase and counterphase pattern. It is found that dual oscillating foils generate higher air flow than a single foil. In addition, it is obviously observed that the flow average velocity for counter-phase pattern is always larger than that for in-phase pattern. As for the effect of distance between two foils, it shows an optimum in the counter-phase pattern. However, the optimum condition does not appear in the inphase pattern. 


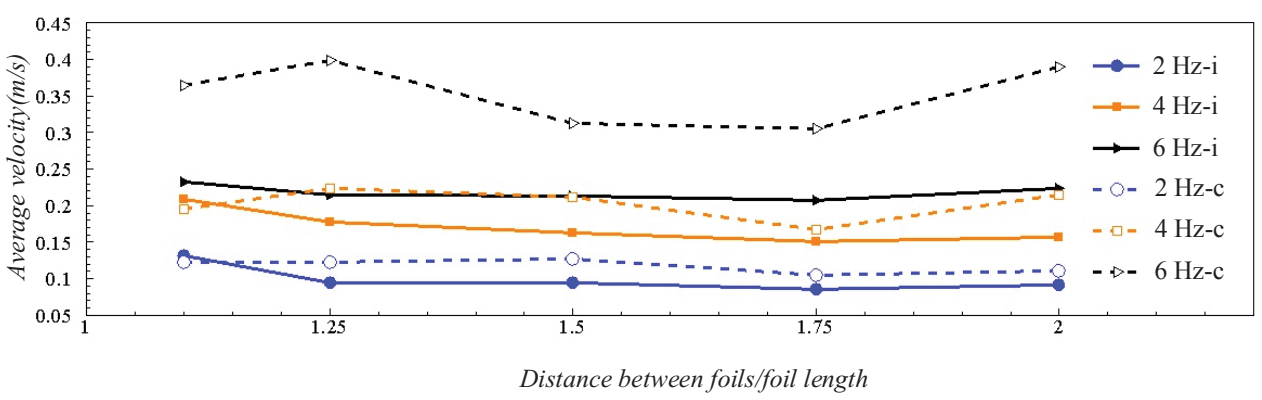

Figure 9. Effect of distance between two foils on the averaged velocity in the channel with three oscillating frequencies for both in-phase and counter-phase pattern.

\section{Conclusions}

In this paper, the air flow driven by oscillating foils in a channel has been numerically studied in detailed. Brief summaries are listed below:

1. Oscillating foils with higher frequencies generate higher flow average velocity than those with lower frequencies.

2. The effect of span angle on the flow average velocity in the channel has been investigated. It is discovered that the optimum flow average velocity occurs at the span angle of $120^{\circ}$.

3. It is discovered that the flow average velocity in the channel is larger than that FFSB pattern with FFSB pattern is larger than that with sinusoidal pattern for three oscillating frequencies.

4. In the oscillating range, it is observed that dual oscillating foils generate higher air flow than single foil. In addition, dual foil with counter-phase oscillating mode produces higher velocity air flow than that with in-phase oscillating mode. Besides, it shows an optimum in the counter-phase oscillating mode. However, the optimum condition does not appear in the in-phase oscillating mode.

\section{Acknowledgement}

The financial support of this research by the Ministry of Science and Technology under the Contract MOST 104-2221-E-149-002, MOST 105-2632-E-131-002, MOST 105-2632-E-131-003 is appreciated. Also, the support from Taipei City University of Science and Technology is acknowledged.

\section{References}

1. M. Toda, Ferroelectrics 22, 911 (1979)

2. M. Toda, Ferroelectrics 32, 127 (1981)

3. J.H. Yoo, J.I. Hong, W. Cao, Sensor Actuator A-Phys 79, 8 (2000)

4. S.M. Wait, S. Basak, S.V. Garimella, A. Raman, IEEE Trans. Compon. Packag. Technol. 30, 119 (2007)

5. T. Acikalin, S.M. Wait, S.V. Garimella, A. Raman, Heat Transfer Eng. 25, 4 (2004)

6. S.F. Liu, R.T. Huang, W.J. Sheu, C.C. Wang, Int. J. Heat Mass Tran. 52, 2565 (2009)

7. M.L. Kimber, S.V. Garimella, Int. J. Heat Mass Tran. 52, pp. 4470 (2009)

8. H.Y. Li, S.M. Chao, J.W. Chen, J.T. Yang, Int. J. Heat Mass Tran. 57, pp. 722 (2013)

9. M. Kimber, S.V. Garimella, ASME J. Heat Tran. 131, 111401 (2009)

10. H.K. Ma, L.K. Tan, Y.T. Li, C.L. Liu, Int. Comm. Heat Tran. Vol. 55, pp. 77 (2014) 